\title{
A Study on Conservative C-Bochner Curvature Tensor in K-Contact and Kenmotsu Manifolds Admitting Semisymmetric Metric Connection
}

\author{
Gurupadavva Ingalahalli and C. S. Bagewadi \\ Department of Mathematics, Kuvempu University, Shankaraghatta-577 451, Shimoga, Karnataka, India \\ Correspondence should be addressed to C. S. Bagewadi, prof_bagewadi@yahoo.co.in
}

Received 30 November 2011; Accepted 18 December 2011

Academic Editors: A. Morozov and C. Qu

Copyright (c) 2012 G. Ingalahalli and C. S. Bagewadi. This is an open access article distributed under the Creative Commons Attribution License, which permits unrestricted use, distribution, and reproduction in any medium, provided the original work is properly cited.

The paper deals with the study on conservative C-Bochner curvature tensor in K-contact and Kenmotsu manifolds admitting semisymmetric metric connection, and it is shown that these manifolds are $\eta$-Einstein with respect to Levi-Civita connection, and the results are illustrated with examples.

\section{Introduction}

In 1924, Friedmann and Schouten [1] introduced the idea of semisymmetric linear connection on a differentiable manifold. In 1932, Hayden [2] introduced the idea of metric connection with torsion on a Riemannian manifold. A systematic study of the semisymmetric metric connection on a Riemannian manifold was published by Yano [3] in 1970. After that the properties of semisymmetric metric connection have been studied by many authors like Amur and Pujar [4], Bagewadi [5], Sharfuddin and Hussain [6], De and Pathak [7], and so forth.

A K-contact manifold is a differentiable manifold with a contact metric structure such that $\xi$ is a Killing vector field $([8,9])$. These are studied by many authors like $([8-11])$. The notion of Kenmotsu manifolds was defined by Kenmotsu [12]. Kenmotsu proved that a locally Kenmotsu manifold is a warped product $I \times_{f} N$ of an interval $I$ and a Kaehler manifold $N$ with warping function $f(t)=s e^{t}$, where $s$ is a nonzero constant. For example it is hyperbolic space $(-1)$. Kenmotsu manifolds were studied by many authors such as Binh et al. [13], Bagewadi and Venkatesha [14]. 
In this paper we study conservative C-Bochner curvature tensor in K-contact and Kenmotsu manifolds admitting semisymmetric metric connection and obtain results on K-contact and Kenmotsu manifolds with respect to Levi-Civita connection.

\section{Preliminaries}

An $n$-dimensional differential manifold $M$ is said to have an almost contact structure $(\phi, \xi, \eta)$ if it carries a tensor field $\phi$ of type $(1,1)$, a vector field $\xi$, and 1-form $\eta$ on $M$, respectively, such that

$$
\phi^{2}=-I+\eta \otimes \xi, \quad \eta(\xi)=1, \quad \eta \circ \phi=0, \quad \phi \xi=0 .
$$

Thus a manifold $M$ equipped with this structure is called an almost contact manifold [9] and is denoted by $(M, \phi, \xi, \eta)$. If $g$ is a Riemannian metric on an almost contact manifold $M$ such that,

$$
g(\phi X, \phi Y)=g(X, Y)-\eta(X) \eta(Y), \quad g(X, \xi)=\eta(X),
$$

where $X$ and $Y$ are vector fields defined on $M$, then $M$ is said to have an almost contact metric structure $(\phi, \xi, \eta, g)$ and $M$ with this structure is called an almost contact metric manifold and is denoted by $(M, \phi, \xi, \eta, g)$.

If on $(M, \phi, \xi, \eta, g)$ the exterior derivative of 1 -form $\eta$ satisfies,

$$
d \eta(X, Y)=g(X, \phi Y)
$$

then $(\phi, \xi, \eta, g)$ is said to be a contact metric structure and $M$ equipped with a contact metric structure is called contact metric manifold.

If moreover $\xi$ is Killing vector field, then $M$ is called a K-contact manifold [8, 9]. A $\mathrm{K}$-contact manifold is called Sasakian [9], if the relation

$$
\left(\nabla_{X} \phi\right) Y=g(X, Y) \xi-\eta(Y) X
$$

holds, where $\nabla$ denotes the operator of covariant connection with respect to $g$.

An almost contact metric manifold, which satisfies the following conditions,

$$
\begin{aligned}
\left(\nabla_{X} \phi\right) Y & =-\eta(Y) \phi X-g(X, \phi Y) \xi \\
\nabla_{X} \xi & =X-\eta(X) \xi
\end{aligned}
$$

where $\nabla$ denotes the Riemannian connection of $g$ hold, $(M, \phi, \xi, \eta, g)$ is called a Kenmotsu manifold. 
In a K-contact manifold $M$, the following properties hold:

$$
\begin{gathered}
\nabla_{X} \xi=-\phi X \\
g(R(\xi, X) Y, \xi)=g(X, Y)-\eta(X) \eta(Y), \\
R(\xi, X) \xi=-X+\eta(X) \xi \\
S(X, \xi)=(n-1) \eta(X)
\end{gathered}
$$

where $R$ is the Riemannian curvature tensor, $S$ is the Ricci tensor and $Q$ is the Ricci operator of $M$, respectively.

In a Kenmotsu manifold $M$, the following properties hold [12]:

$$
\begin{aligned}
\nabla_{X} \xi & =X-\eta(X) \xi, \\
R(X, Y) \xi & =\eta(X) Y-\eta(Y) X, \\
S(X, \xi) & =-(n-1) \eta(X), \\
Q \xi & =-(n-1) \xi,
\end{aligned}
$$

where $R$ is the Riemannian curvature tensor, $S$ is the Ricci tensor, and $Q$ is the Ricci operator of $M$, respectively.

Let $M$ be an $n$-dimensional Riemannian manifold of class $C^{\infty}$ with metric tensor $g$ and let $\nabla$ be the Levi-Civita connection on $M$. A linear connection $\tilde{\nabla}$ in an almost contact metric manifold $M$ is said to be a semisymmetric connection if the torsion tensor $T$ of the connection $\tilde{\nabla}$ satisfies

$$
T(X, Y)=\pi(Y) X-\pi(X) Y,
$$

where $\pi$ is a 1 -form on $M$ with $\rho$ as associated vector field, that is, $\pi(X)=g(X, \rho)$ for any differentiable vector field $X$ on $M$.

A semisymmetric connection $\tilde{\nabla}$ is called semisymmetric metric connection if it further satisfies $\tilde{\nabla} g=0$.

In an almost contact manifold semisymmetric metric connection is defined by identifying the 1 -form of (2.14) with the contact-form $\eta$, that is,

$$
T(X, Y)=\eta(Y) X-\eta(X) Y,
$$

with $\xi$ as associated vector field, that is, $g(X, \xi)=\eta(X)$.

The relation between the semisymmetric metric connection $\tilde{\nabla}$ and the Levi-Civita connection $\nabla$ of $M$ has been obtained by Yano [3], which is given by

$$
\tilde{\nabla}_{X} Y=\nabla_{X} Y+\eta(Y) X-g(X, Y) \xi \text {. }
$$

The above condition satisfies K-contact and Kenmotsu manifolds also. 
We denote $R, S$, and $r$ by curvature tensor, Ricci tensor, and scalar curvature with respect to Levi-Civita connection and correspondingly $\widetilde{R}, \widetilde{S}$, and $\widetilde{r}$ with respect to semisymmetric metric connection. If $B$ denotes C-Bochner curvature tensor [15] with respect to LeviCivita connection $\widetilde{B}$ with respect to semisymmetric metric connection is given by

$$
\begin{aligned}
& \widetilde{B}(X, Y) Z=\widetilde{R}(X, Y) Z+\frac{1}{n+3} \\
& \times[g(X, Z) \widetilde{Q} Y-\widetilde{S}(Y, Z) X-g(Y, Z) \widetilde{Q} X+\widetilde{S}(X, Z) Y \\
&+g(\phi X, Z) \widetilde{Q} \phi Y-\widetilde{S}(\phi Y, Z) \phi X-g(\phi Y, Z) \widetilde{Q} \phi X+\widetilde{S}(\phi X, Z) \phi Y \\
&+2 \widetilde{S}(\phi X, Y) \phi Z+2 g(\phi X, Y) \widetilde{Q} \phi Z+\eta(Y) \eta(Z) \widetilde{Q} X-\eta(Y) \widetilde{S}(X, Z) \xi \\
&+\eta(X) \widetilde{S}(Y, Z) \xi-\eta(X) \eta(Z) \widetilde{Q} Y] \\
&- \frac{\tilde{D}+n-1}{n+3}[g(\phi X, Z) \phi Y-g(\phi Y, Z) \phi X+2 g(\phi X, Y) \phi Z] \\
&+ \frac{\tilde{D}}{n+3}[\eta(Y) g(X, Z) \xi-\eta(Y) \eta(Z) X+\eta(X) \eta(Z) Y-\eta(X) g(Y, Z) \xi] \\
&-\frac{\widetilde{D}-4}{n+3}[g(X, Z) Y-g(Y, Z) X],
\end{aligned}
$$

where $\widetilde{D}=(n-1+\widetilde{r}) /(n+1)$.

Differentiate (2.17) covariantly with respect to $\tilde{\nabla}$ and then contracting we get

$$
\begin{aligned}
& (\operatorname{Div} \cdot \widetilde{B})=\frac{n+2}{n+3}\left[\left(\tilde{\nabla}_{X} \widetilde{S}\right)(Y, Z)-\left(\widetilde{\nabla}_{Y} \widetilde{S}\right)(X, Z)\right]+\frac{1}{n+3} \\
& \times\left[\left(\tilde{\nabla}_{\phi Y} \widetilde{S}\right)(\phi X, Z)-\left(\widetilde{\nabla}_{\phi X} \widetilde{S}\right)(\phi Y, Z)+2\left(\tilde{\nabla}_{\phi Z} \widetilde{S}\right)(\phi X, Y)-\left(\widetilde{\nabla}_{\xi} \widetilde{S}\right)(X, Z) \eta(Y)\right. \\
& \left.-\widetilde{S}(X, Z)\left(\tilde{\nabla}_{\xi} \eta\right)(Y)-\left(\tilde{\nabla}_{\xi} \tilde{S}\right)(Y, Z) \eta(X)+\widetilde{S}(Y, Z)\left(\tilde{\nabla}_{\xi} \eta\right)(X)\right] \\
& +\frac{(n-1)}{2(n+1)(n+3)}\left[\left(\tilde{\nabla}_{Y} \tilde{r}\right) g(\phi X, \phi Z)-\left(\tilde{\nabla}_{X} \tilde{r}\right) g(\phi Y, \phi Z)\right] \\
& +\frac{n}{(n+1)(n+3)}\left[\left(\tilde{\nabla}_{\phi Y} \tilde{r}\right) g(\phi X, Z)-\left(\tilde{\nabla}_{\phi X} \tilde{r}\right) g(\phi Y, Z)+2\left(\tilde{\nabla}_{\phi Z} \tilde{r}\right) g(\phi X, Y)\right] \\
& -\frac{\tilde{r}+(n-1)(n+2)}{(n+1)(n+3)}\left[\eta\left(\tilde{\nabla}_{\phi X} \xi\right) g(\phi Y, Z)-\eta\left(\tilde{\nabla}_{\phi Y} \xi\right) g(\phi X, Z)-2 \eta\left(\tilde{\nabla}_{\phi Z} \xi\right) g(\phi X, Y)\right] \\
& +\frac{\tilde{r}+(n-1)}{(n+1)(n+3)}\left[\left(\tilde{\nabla}_{\xi} \eta\right)(Y) g(X, Z)-\left(\tilde{\nabla}_{\xi} \eta\right)(X) g(Y, Z)+\left(\tilde{\nabla}_{Y} \eta\right)(Z) \eta(X)\right. \\
& \left.-\left(\tilde{\nabla}_{X} \eta\right)(Z) \eta(Y)+\left\{\left(\tilde{\nabla}_{Y} \eta\right)(X)-\left(\tilde{\nabla}_{X} \eta\right)(Y)\right\} \eta(Z)\right] \\
& +\frac{\left(\tilde{\nabla}_{\xi} \tilde{r}\right)}{(n+1)(n+3)}[g(X, Z) \eta(Y)-g(Y, Z) \eta(X)] \text {. }
\end{aligned}
$$




\section{Relation between $R, S_{r} r$ and $\widetilde{R}, \widetilde{S}, \widetilde{r}$ in a K-Contact Manifold}

A relation between the curvature tensor $R$ and $\widetilde{R}$ of type $(1,3)$ of the connections $\nabla$ and $\tilde{\nabla}$ by using (2.16) is given by

$$
\begin{aligned}
\tilde{R}(X, Y) Z= & R(X, Y) Z+[g(\phi Y, Z) X-g(\phi X, Z) Y]+[g(Y, Z) \phi X-g(X, Z) \phi Y] \\
& +[g(\phi X, \phi Z) Y-g(\phi Y, \phi Z) X]+[g(Y, Z) \eta(X)-g(X, Z) \eta(Y)] \xi
\end{aligned}
$$

From (3.1), it follows that

$$
\widetilde{S}(Y, Z)=S(Y, Z)+(n-2) g(\phi Y, Z)-(n-2) g(Y, Z)+(n-2) \eta(Y) \eta(Z)
$$

where $\widetilde{S}$ denotes the Ricci tensor with respect to semisymmetric metric connection and $S$ denotes the Ricci tensor. On contacting (3.2), we get

$$
\tilde{r}=r-(n-1)(n-2),
$$

where $\tilde{r}$ and $r$ are scalar curvatures with respect to semisymmetric metric connection and Levi-Civita connection.

In a Riemannian manifold $M, \xi$ is a Killing vector field in K-contact manifold, that is, $S$ and $r$ are invariant under it, that is,

$$
\begin{gathered}
\left(L_{\xi} g\right)(X, Y)=g\left(\nabla_{X} \xi, Y\right)+g\left(X, \nabla_{Y} \xi\right)=0, \\
\left(L_{\xi} S\right)(X, Y)=\left(L_{\xi} g\right)(Q X, Y)=0, \\
\left(L_{\xi} r\right)=0
\end{gathered}
$$

where $L$ is Lie-derivative. We know that

$$
\begin{aligned}
\left(\nabla_{\xi} S\right)(Y, Z) & =\xi S(Y, Z)-S\left(\nabla_{\xi} Y, Z\right)-S\left(Y, \nabla_{\xi} Z\right) \\
& =\left(L_{\xi} S\right)(Y, Z)-S\left(\nabla_{Y} \xi, Z\right)-S\left(Y, \nabla_{Z} \xi\right)
\end{aligned}
$$

by using (3.5) in (3.7) and by virtue of (2.6), we have

$$
\left(\nabla_{\xi} S\right)(Y, Z)=0
$$


Now in a K-contact manifold $\tilde{L}, \widetilde{S}$, and $\tilde{r}$ are with respect to semisymmetric metric connection, that is,

$$
\begin{gathered}
\left(\widetilde{L}_{\xi} g\right)(X, Y)=2[g(X, Y)-\eta(X) \eta(Y)] \neq 0, \\
\left(\widetilde{L}_{\xi} \tilde{S}\right)(X, Y)=2(n-2) g(\phi X, Y)+2 g(X, Y)-2 \eta(X) \eta(Y) \neq 0, \\
\left(\tilde{L}_{\tilde{\xi}} \tilde{r}\right) \neq 0 .
\end{gathered}
$$

This shows that it is not $\mathrm{K}$-contact with respect to a semisymmetric metric connection.

Now from (3.2), we have

$$
\begin{aligned}
\left(\tilde{\nabla}_{W} \tilde{S}\right)(Y, Z)= & \left(\nabla_{W} S\right)(Y, Z)+2(n-2) g(Y, W) \eta(Z)-2(n-2) \eta(Y) g(Z, \phi W) \\
& -2(n-2) \eta(Y) \eta(Z) \eta(W)-\eta(Y) S(W, Z)-\eta(Z) S(Y, W) \\
& +(n-1) g(W, Y) \eta(Z)+(n-1) g(W, Z) \eta(Y)
\end{aligned}
$$

Put $W=\xi$ in (3.10), we have

$$
\begin{aligned}
\left(\tilde{\nabla}_{\xi} \tilde{S}\right)(Y, Z) & =\left(\nabla_{\xi} S\right)(Y, Z) \\
\left(\tilde{\nabla}_{\xi} \tilde{r}\right) & =\left(\nabla_{\xi} r\right)
\end{aligned}
$$

But

$$
\begin{gathered}
\left(\nabla_{\xi} S\right)(Y, Z)=0 \Longrightarrow\left(\tilde{\nabla}_{\xi} \tilde{S}\right)(Y, Z)=0, \\
\left(\nabla_{\xi} r\right)=0 \Longrightarrow\left(\tilde{\nabla}_{\xi} \tilde{r}\right)=0 .
\end{gathered}
$$

Put $Z=\xi$ in (3.10), we have

$$
\begin{aligned}
\left(\tilde{\nabla}_{W} \tilde{S}\right)(Y, \xi)= & S(Y, \phi W)-S(Y, W)+(n-1) g(Y, W)-(n-1) g(Y, \phi W) \\
& +2(n-2) g(\phi Y, \phi W)
\end{aligned}
$$




\section{K-Contact Manifold Admitting Semisymmetric Metric Connection with Div $\cdot \widetilde{B}=0$}

Considering $\operatorname{Div} \cdot \widetilde{B}=0$ in (2.18) and putting $X=\xi$ in the equation; using (3.2), (3.3), (3.12), (3.13), and (3.14) and by virtue of (2.1), (2.6), and (2.16), we get

$$
\begin{aligned}
& \frac{n+2}{n+3}[-S(\phi Y, Z)+(3 n-5) g(Z, \phi Y)+S(Y, Z)-(n-1) g(Y, Z)] \\
& \quad-\frac{(n-1)}{2(n+1)(n+3)}\left[\left(\nabla_{\xi} r\right) g(\phi Y, \phi Z)\right]+\frac{r-(n-1)(n-3)}{(n+1)(n+3)}[g(Y, \phi Z)+g(Y, Z)-\eta(Y) \eta(Z)] \\
& \quad+\frac{\left(\nabla_{\xi} r\right)}{(n+1)(n+3)}[\eta(Y) \eta(Z)-g(Y, Z)]=0 .
\end{aligned}
$$

In a K-contact manifold, $\xi[r]=0$, that is, $\nabla_{\xi} r=0$ then the above equation reduces to

$$
\begin{gathered}
\frac{n+2}{n+3}[-S(\phi Y, Z)+(3 n-5) g(Z, \phi Y)+S(Y, Z)-(n-1) g(Y, Z)] \\
+\frac{r-(n-1)(n-3)}{(n+1)(n+3)}[g(Y, \phi Z)+g(Y, Z)-\eta(Y) \eta(Z)]=0 .
\end{gathered}
$$

Interchanging $Y$ and $Z$ in the above equation then we have

$$
\begin{gathered}
\frac{n+2}{n+3}[-S(\phi Z, Y)+(3 n-5) g(Y, \phi Z)+S(Z, Y)-(n-1) g(Z, Y)] \\
+\frac{r-(n-1)(n-3)}{(n+1)(n+3)}[g(Z, \phi Y)+g(Z, Y)-\eta(Y) \eta(Z)]=0
\end{gathered}
$$

Adding these equation (4.2) and (4.3), we have

$$
S(Y, Z)=\left[\frac{(n-1)\left(n^{2}+4 n-1\right)-r}{(n+1)(n+2)}\right] g(Y, Z)+\left[\frac{r-(n-1)(n-3)}{(n+1)(n+2)}\right] \eta(Y) \eta(Z)
$$

Then the above equation is written as $S(Y, Z)=\alpha g(Y, Z)+\beta \eta(Y) \eta(Z)$, where

$$
\alpha=\left[\frac{(n-1)\left(n^{2}+4 n-1\right)-r}{(n+1)(n+2)}\right], \quad \beta=\left[\frac{r-(n-1)(n-3)}{(n+1)(n+2)}\right]
$$


On contracting (4.4), we get

$$
R_{1}=\frac{(n-1)\left[n\left(n^{2}+4 n-1\right)-r-(n-3)\right]}{(n+1)(n+2)} .
$$

Hence we state the following theorem.

Theorem 4.1. If in a K-contact manifold the C-Bochner curvature tensor with respect to semisymmetric metric connection is conservative, then the manifold is $\eta$-Einstein with respect to Levi-Civita connection and the scalar curvature of such a manifold is given in (4.6).

\section{Example for K-Contact Manifold}

Consider the 3-dimensional manifold $M=\left\{(x, y, z):(x, y, z) \in R^{3}\right\}$, where $(x, y, z)$ are the standard coordinates in $R^{3}$. Let $\left(E_{1}, E_{2}, E_{3}\right)$ be linearly independent at each point of $M$

$$
E_{1}=\frac{\partial}{\partial x}+y \frac{\partial}{\partial z}, \quad E_{2}=\frac{\partial}{\partial y}-x \frac{\partial}{\partial z}, \quad E_{3}=\frac{\partial}{\partial z}
$$

Let $g$ be the Riemannian metric defined by

$$
\begin{aligned}
& g\left(E_{1}, E_{2}\right)=g\left(E_{2}, E_{3}\right)=g\left(E_{1}, E_{3}\right)=0, \\
& g\left(E_{1}, E_{1}\right)=g\left(E_{2}, E_{2}\right)=g\left(E_{3}, E_{3}\right)=1,
\end{aligned}
$$

where $g$ is given by $g=\left[\left(1-y^{2}\right) d x \otimes d x+\left(1-x^{2}\right) d y \otimes d y+d z \otimes d z\right]$. Let $\xi$ be the vector field, $\eta$ be the 1 -form, and $\phi$ be the $(1,1)$ tensor field defined by

$$
\begin{aligned}
\xi & =\frac{\partial}{\partial z}, \quad \eta=d z+x d y-y d x, \\
\phi E_{1} & =-E_{2}, \quad \phi E_{2}=E_{1}, \quad \phi E_{3}=0 .
\end{aligned}
$$

The linearity property of $\phi$ and $g$ yields that

$$
\begin{gathered}
\eta\left(E_{3}\right)=1, \quad \phi^{2} U=-U+\eta(U) E_{3} \\
g(\phi U, \phi W)=g(U, W)-\eta(U) \eta(W)
\end{gathered}
$$

for any vector fields $U, W$ on $M$. Thus for $E_{3}=\xi$. The structure $(\phi, \xi, \eta, g)$ defines on $M$. By definition of Lie bracket, we have

$$
\left[E_{1}, E_{2}\right]=-2 E_{3}, \quad\left[E_{1}, E_{3}\right]=\left[E_{2}, E_{3}\right]=0
$$


Let $\nabla$ be Levi-Civita connection with respect to the above metric $g$ given by Koszul formula, that is

$$
\begin{aligned}
2 g\left(\nabla_{X} Y, Z\right)= & X(g(Y, Z))+Y(g(Z, X))-Z(g(X, Y)) \\
& -g(X,[Y, Z])-g(Y,[X, Z])+g(Z,[X, Y]) .
\end{aligned}
$$

Then by Koszula formula, we have

$$
\begin{array}{ccc}
\nabla_{E_{1}} E_{1}=0, & \nabla_{E_{2}} E_{2}=0, & \nabla_{E_{3}} E_{3}=0, \\
\nabla_{E_{1}} E_{2}=-E_{3}, & \nabla_{E_{2}} E_{1}=-E_{3}, & \nabla_{E_{2}} E_{3}=-E_{1}, \\
\nabla_{E_{1}} E_{3}=E_{2}, & \nabla_{E_{3}} E_{1}=E_{2}, & \nabla_{E_{3}} E_{2}=-E_{1} .
\end{array}
$$

Clearly one can see that $(\phi, \xi, \eta, g)$ is a K-contact structure.

The Ricci tensor $S(X, Y)$ is given by

$$
\begin{aligned}
S(X, Y) & =\sum_{i=1}^{3} g\left(R\left(X, E_{i}\right) E_{i}, Y\right) \\
& =g\left(R\left(X, E_{1}\right) E_{1}, Y\right)+g\left(R\left(X, E_{2}\right) E_{2}, Y\right)+g\left(R\left(X, E_{3}\right) E_{3}, Y\right) .
\end{aligned}
$$

The nonzero components of $R\left(X, E_{i}\right) E_{i}$, where $i=1,2,3$, and by virtue of (5.7) we have

$$
\begin{array}{lc}
R\left(E_{2}, E_{1}\right) E_{1}=-3 E_{2}, & R\left(E_{3}, E_{1}\right) E_{1}=E_{3}, \\
R\left(E_{1}, E_{2}\right) E_{2}=-3 E_{1}, & R\left(E_{3}, E_{2}\right) E_{2}=E_{3}, \\
R\left(E_{1}, E_{3}\right) E_{3}=E_{1}, & R\left(E_{2}, E_{3}\right) E_{3}=E_{2} .
\end{array}
$$

Using these in (5.8), we have

$$
S(X, Y)=-2 g(X, Y)+4 \eta(X) \eta(Y)
$$

This shows that $R^{3}$ is an $\eta$-Einstein. This is an example of $\mathrm{K}$-contact manifold which is an $\eta$-Einstein.

If $X=Y=Z=E_{i}$, in (2.18) and by virtue of (3.2), we obtain (Div $\left.\widetilde{B}\right)=0$. Thus Theorem 4.1 holds true.

However, if $X \neq Y \neq Z=E_{i}$, in (2.18) and by virtue of (3.2), we obtain (Div $\left.\cdot \widetilde{B}\right) \neq 0$. Hence in general, if $X=\sum_{i=1}^{3} a_{i} E_{i}, Y=\sum_{i=1}^{3} b_{i} E_{i}$ and $Z=\sum_{i=1}^{3} c_{i} E_{i}$, then $(\operatorname{Div} \cdot \widetilde{B})(X, Y) Z \neq 0$. In this case the converse of Theorem 4.1 does not hold true. 


\section{Relation between $R, S, r$ and $\widetilde{R}, \widetilde{S}, \widetilde{r}$ in a Kenmotsu Manifold}

A relation between the curvature tensor $R$ and $\widetilde{R}$ of type $(1,3)$ of the connections $\nabla$ and $\tilde{\nabla}$ by using (2.16) is given by

$$
\begin{aligned}
\tilde{R}(X, Y) Z= & R(X, Y) Z+[g(X, Z) Y-g(Y, Z) X]+2[g(\phi X, \phi Z) Y-g(\phi Y, \phi Z) X] \\
& +2[g(Y, Z) \eta(X)-g(X, Z) \eta(Y)] \xi .
\end{aligned}
$$

From (6.1), it follows that

$$
\widetilde{S}(Y, Z)=S(Y, Z)-(n-1) g(Y, Z)-2(n-2) g(\phi Y, \phi Z),
$$

where $\widetilde{S}$ denotes the Ricci tensor with respect to semisymmetric metric connection and $S$ denotes the Ricci tensor. On contacting (6.2), we get

$$
\tilde{r}=r-(n-1)(3 n-4),
$$

where $\tilde{r}$ and $r$ are scalar curvatures with respect to semisymmetric metric connection and Levi-Civita connection.

In a Kenmotsu manifold $M, \xi$ is a unit vector field in Kenmotsu manifold, then the following properties hold

$$
\begin{aligned}
\left(L_{\xi} g\right)(X, Y) & =2[g(X, Y)-\eta(X) \eta(Y)], \\
\left(L_{\xi} S\right)(X, Y) & =2[S(X, Y)+(n-1) \eta(X) \eta(Y)], \\
\left(L_{\xi} r\right) & =(\operatorname{Div} \xi) r,
\end{aligned}
$$

where $L$ is a Lie derivative. We know that

$$
\begin{aligned}
\left(\nabla_{\xi} S\right)(Y, Z) & =\xi S(Y, Z)-S\left(\nabla_{\xi} Y, Z\right)-S\left(Y, \nabla_{\xi} Z\right) \\
& =\left(L_{\xi} S\right)(Y, Z)-S\left(\nabla_{Y} \xi, Z\right)-S\left(Y, \nabla_{Z} \xi\right) .
\end{aligned}
$$

By using (6.4) in (6.5) and by virtue of (2.10) we have

$$
\begin{gathered}
\left(\nabla_{\xi} S\right)(Y, Z)=0, \\
\left(\nabla_{\xi} r\right)=0 .
\end{gathered}
$$


Now in a Kenmotsu manifold $\widetilde{L}, \widetilde{S}$, and $\widetilde{r}$ are with respect to semisymmetric metric connection, then we define the properties like

$$
\begin{aligned}
&\left(\widetilde{L}_{\xi} g\right)(X, Y)=4[g(X, Y)-\eta(X) \eta(Y)], \\
&\left(\widetilde{L}_{\xi} \widetilde{S}\right)(X, Y)=4[ S(X, Y)-(n-1) g(\phi X, \phi Y)-2(n-2) g(\phi X, \phi Y) \\
&+(n-1) \eta(X) \eta(Y)], \\
& \tilde{L}_{\xi} \tilde{r}=(\operatorname{Div} \tilde{\xi}) \tilde{r} .
\end{aligned}
$$

Now from (6.2), we have

$$
\begin{aligned}
\left(\tilde{\nabla}_{X} \tilde{S}\right)(Y, Z)= & \left(\nabla_{X} S\right)(Y, Z)-(n-1)[g(X, Y) \eta(Z)+g(X, Z) \eta(Y)] \\
& -[\eta(Y) S(X, Z)+\eta(Z) S(X, Y)]+4(n-2) \\
& \times[\eta(Y) g(\phi X, \phi Z)+g(\phi X, \phi Y) \eta(Z)] .
\end{aligned}
$$

Putting $X=\xi$ in the above equation and by virtue of (6.6) then we have

$$
\begin{gathered}
\left(\widetilde{\nabla}_{\xi} \widetilde{S}\right)(Y, Z)=0, \\
\left(\tilde{\nabla}_{\xi} \tilde{r}\right)=0 .
\end{gathered}
$$

Again putting $Z=\xi$ in (6.9), we have

$$
\left(\tilde{\nabla}_{X} \tilde{S}\right)(Y, \xi)=-2 S(Y, X)-2(n-1) g(Y, X)+4(n-2) g(\phi Y, \phi X)
$$

\section{Kenmotsu Manifold Admitting Semisymmetric Metric Connection with Div $\cdot \widetilde{B}=0$}

Considering Div $\cdot \widetilde{B}=0$ in (2.18) and putting $X=\xi$ in the equation; using (6.2), (6.3), (6.10), and (6.12) and by virtue of (2.10), (2.16) and on simplification we get

$$
\begin{aligned}
& \frac{n+2}{n+3}[2 S(Y, Z)+2(n-1) g(Y, Z)-4(n-2) g(\phi Y, \phi Z)] \\
& \quad-\frac{(n-1)}{2(n+1)(n+3)}\left[\left(\nabla_{\xi} r\right) g(\phi Y, \phi Z)\right]+\frac{r-(n-1)(3 n-4)+(n-1)}{(n+1)(n+3)} \\
& \quad \times[2 g(Y, Z)-2 \eta(Y) \eta(Z)]-\frac{\left(\nabla_{\xi} r\right)}{(n+1)(n+3)}[g(\phi Y, \phi Z)]=0
\end{aligned}
$$


In a Kenmotsu manifold $M, \nabla_{\xi} r=0$ then the above equation reduces to

$$
\begin{gathered}
\frac{n+2}{n+3}[2 S(Y, Z)+2(n-1) g(Y, Z)-4(n-2) g(\phi Y, \phi Z)] \\
+\frac{r-(n-1)(3 n-5)}{(n+1)(n+3)}[2 g(Y, Z)-2 \eta(Y) \eta(Z)]=0 .
\end{gathered}
$$

On simplifying the above equation we get

$$
\begin{aligned}
S(Y, Z)= & {\left[(n-3)-\frac{r-(n-1)(3 n-5)}{(n+1)(n+2)}\right] g(Y, Z) } \\
& +\left[-2(n-2)+\frac{r-(n-1)(3 n-5)}{(n+1)(n+2)}\right] \eta(Y) \eta(Z) .
\end{aligned}
$$

Then the above equation is written as $S(Y, Z)=\alpha g(Y, Z)+\beta \eta(Y) \eta(Z)$, where

$$
\alpha=\left[(n-3)-\frac{r-(n-1)(3 n-5)}{(n+1)(n+2)}\right], \quad \beta=\left[-2(n-2)+\frac{r-(n-1)(3 n-5)}{(n+1)(n+2)}\right]
$$

On contracting (7.3), we get

$$
r_{1}=\left[(n-4)(n-1)-\frac{(n-1)[r-(n-1)(3 n-5)]}{(n+1)(n+2)}\right] .
$$

Hence we state the following theorem.

Theorem 7.1. If in a Kenmotsu manifold the C-Bochner curvature tensor with respect to semisymmetric metric connection is conservative, then the manifold is $\eta$-Einstein with respect to Levi-Civita connection, and the scalar curvature of such a manifold is given in (7.5).

\section{Example for Kenmotsu Manifold}

Let $M=\left\{(x, y, z) \in R^{3}\right\}$. Let $\left(E_{1}, E_{2}, E_{3}\right)$ be linearly independent vector fields given by

$$
E_{1}=e^{-z}\left(\frac{\partial}{\partial x}+\frac{\partial}{\partial y}\right), \quad E_{2}=e^{-z}\left(-\frac{\partial}{\partial x}+\frac{\partial}{\partial y}\right), \quad E_{3}=\frac{\partial}{\partial z}
$$

Let $g$ be the Riemannian metric defined by

$$
\begin{aligned}
& g\left(E_{1}, E_{2}\right)=g\left(E_{2}, E_{3}\right)=g\left(E_{1}, E_{3}\right)=0, \\
& g\left(E_{1}, E_{1}\right)=g\left(E_{2}, E_{2}\right)=g\left(E_{3}, E_{3}\right)=1,
\end{aligned}
$$


where $g$ is given by $g=\left(e^{2 z} / 2\right)(d x \otimes d x+d y \otimes d y)+d z \otimes d z$. Let $\xi$ be the vector field, $\eta$ be the 1 -form, and $\phi$ be the $(1,1)$ tensor field defined by

$$
\begin{gathered}
\eta=d z, \quad \xi=E_{3}=\frac{\partial}{\partial z}, \\
\phi E_{1}=E_{2}, \quad \phi E_{2}=-E_{1}, \quad \phi E_{3}=0 .
\end{gathered}
$$

The linearity property of $\phi$ and $g$ yields that

$$
\begin{gathered}
\eta\left(E_{3}\right)=1, \quad \phi^{2} U=-U+\eta(U) E_{3} \\
g(\phi U, \phi W)=g(U, W)-\eta(U) \eta(W)
\end{gathered}
$$

for any vector fields $U, W$ on $M$. By definition of Lie bracket, we have

$$
\left[E_{1}, E_{2}\right]=0, \quad\left[E_{2}, E_{3}\right]=E_{2}, \quad\left[E_{1}, E_{3}\right]=E_{1} .
$$

Let $\nabla$ be the Levi-Civita connection with respect to above metric $g$ which is given by Koszula formula (5.6), and by virtue of it we have

$$
\begin{array}{lrl}
\nabla_{E_{1}} E_{3}=E_{1}, & \nabla_{E_{2}} E_{3}=E_{2}, & \nabla_{E_{3}} E_{3}=0, \\
\nabla_{E_{1}} E_{2}=0, & \nabla_{E_{2}} E_{2}=-E_{3}, & \nabla_{E_{3}} E_{2}=0, \\
\nabla_{E_{1}} E_{1}=-E_{3}, & \nabla_{E_{2}} E_{1}=0, & \nabla_{E_{3}} E_{1}=0 .
\end{array}
$$

Clearly one can see that $(\phi, \xi, \eta, g)$ is a Kenmotsu structure.

The nonzero components of $R\left(X, E_{i}\right) E_{i}$, where $i=1,2,3$, and by virtue of (8.6) we have

$$
\begin{array}{ll}
R\left(E_{1}, E_{2}\right) E_{2}=-E_{1}, & R\left(E_{1}, E_{3}\right) E_{3}=-E_{1}, \\
R\left(E_{2}, E_{1}\right) E_{1}=-E_{2}, & R\left(E_{2}, E_{3}\right) E_{3}=-E_{2}, \\
R\left(E_{3}, E_{1}\right) E_{1}=-E_{3}, & R\left(E_{3}, E_{2}\right) E_{2}=-E_{3} .
\end{array}
$$

The Ricci tensor $S(X, Y)$ is given in (5.8) by virtue of $(8.7)$, and we have

$$
S(X, Y)=-2 g(X, Y)+0 \cdot \eta(X) \eta(Y) .
$$

This shows that $R^{3}$ is an $\eta$-Einstein. This is an example of Kenmotsu manifold which is an $\eta$-Einstein.

If $X=Y=Z=E_{i}$, in (2.18) and by virtue of $(6.2)$, we obtain (Div $\left.\cdot \widetilde{B}\right)=0$. Thus Theorem 7.1 holds true.

However, if $X \neq Y \neq Z=E_{i}$, in (2.18) and by virtue of (6.2), we obtain (Div $\left.\widetilde{B}\right) \neq 0$. Hence in general, if $X=\sum_{i=1}^{3} a_{i} E_{i}, Y=\sum_{i=1}^{3} b_{i} E_{i}$ and $Z=\sum_{i=1}^{3} c_{i} E_{i}$, then $(\operatorname{Div} \cdot \widetilde{B})(X, Y) Z \neq 0$. In this case the converse of Theorem 7.1 does not hold true. 


\section{Acknowledgments}

The authors express their gratitude to DST (Department of Science and Technology), Government of India for providing financial assistance under major Research Project (No. SR/S4/MS: 482/07). They are grateful to referees for revising the paper.

\section{References}

[1] A. Friedmann and J. A. Schouten, "Über die geometrie der halbsymmetrischen Übertragungen," Mathematische Zeitschrift, vol. 21, no. 1, pp. 211-223, 1924.

[2] H. A. Hayden, "Subspaces of a space with torsion," Proceedings of the London Mathematical Society, vol. 34, pp. 27-50, 1932.

[3] K. Yano, "On semi-symmetric metric connection," Revue Roumaine de Mathématiques Pures et Appliquées, vol. 15, pp. 1579-1586, 1970.

[4] K. S. Amur and S. S. Pujar, “On submanifolds of a Riemannian manifold admitting a metric semisymmetric connection," The Tensor Society. Tensor. New Series, vol. 32, no. 1, pp. 35-38, 1978.

[5] C. S. Bagewadi, "On totally real submanifolds of a Kahlerian manifold admitting semi-symmetric metric F-connection," Indian Journal of Pure and Applied Mathematics, vol. 13, no. 5, pp. 528-536, 1982.

[6] A. Sharfuddin and S. I. Husain, "Semi-symmetric metric connexions in almost contact manifolds," The Tensor Society. Tensor. New Series, vol. 30, no. 2, pp. 133-139, 1976.

[7] U. C. De and G. Pathak, "On a semi-symmetric metric connection in a Kenmotsu manifold," Bulletin of the Calcutta Mathematical Society, vol. 94, no. 40, pp. 319-324, 2002.

[8] S. Sasaki, Lecture Note on almost Contact Manifolds, Tohoku University, Tohoku, Japan, 1965.

[9] D. E. Blair, Contact Manifolds in Riemannian Geometry, vol. 509 of Lecture Notes in Mathematics, Springer, Berlin, Germany, 1976.

[10] M. M. Tripathi and M. K. Dwivedi, "The structure of some classes of K-contact manifolds," Proceedings of the Indian Academy of Sciences: Mathematical Sciences, vol. 118, no. 3, pp. 371-379, 2008.

[11] K. T. Pradeep Kumar, C. S. Bagewadi, and Venkatesha, "Projective $\phi$-symmetric K-contact manifold admitting quarter-symmetric metric connection," Differential Geometry: Dynamical Systems, vol. 13, pp. 128-137, 2011.

[12] K. Kenmotsu, "A class of almost contact Riemannian manifolds," The Tohoku Mathematical Journal, vol. 24, pp. 93-103, 1972.

[13] T. Q. Binh, L. Tamássy, U. C. De, and M. Tarafdar, "Some remarks on almost Kenmotsu manifolds," Mathematica Pannonica, vol. 13, no. 1, pp. 31-39, 2002.

[14] C. S. Bagewadi and Venkatesha, "Some curvature tensors on a Kenmotsu manifold," The Tensor Society. Tensor. New Series, vol. 68, no. 2, pp. 140-147, 2007.

[15] J.-S. Kim, M. M. Tripathi, and J. Choi, "On C-Bochner curvature tensor of a contact metric manifold," Bulletin of the Korean Mathematical Society, vol. 42, no. 4, pp. 713-724, 2005. 


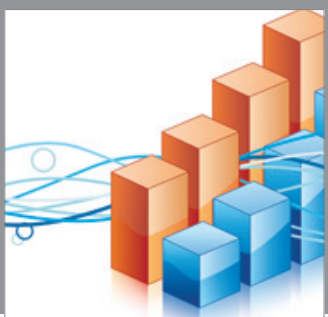

Advances in

Operations Research

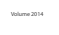

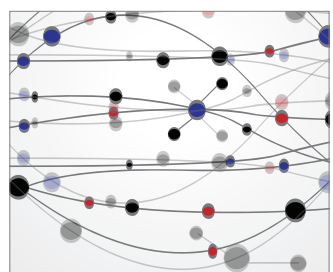

\section{The Scientific} World Journal
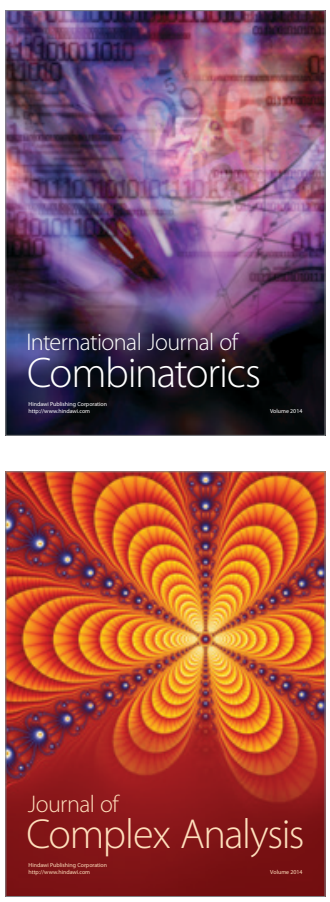

International Journal of

Mathematics and

Mathematical

Sciences
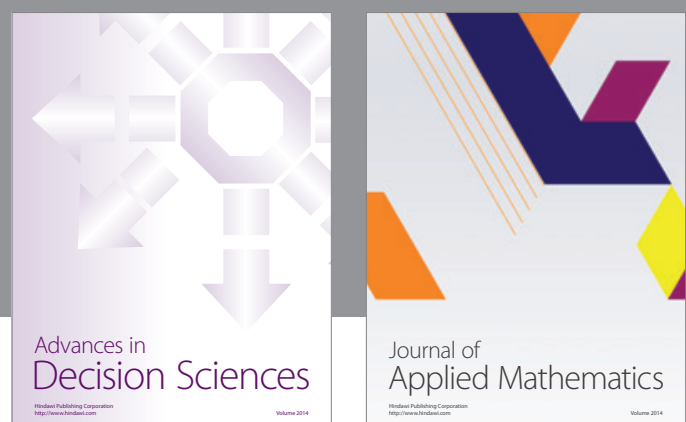

Journal of

Applied Mathematics
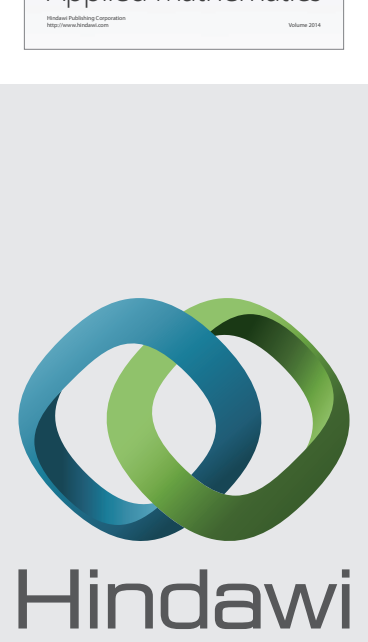

Submit your manuscripts at http://www.hindawi.com
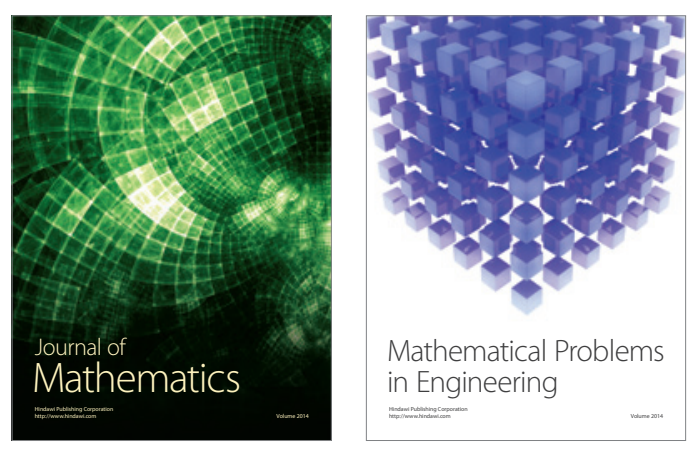

Mathematical Problems in Engineering
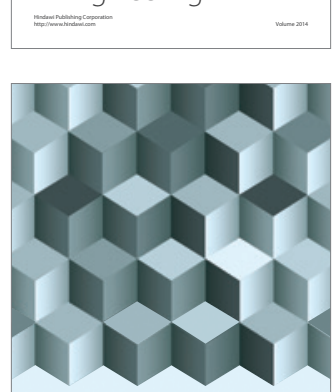

Journal of

Function Spaces
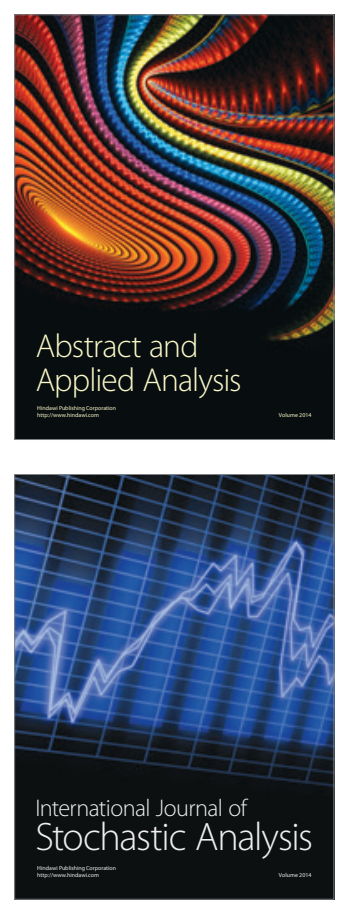

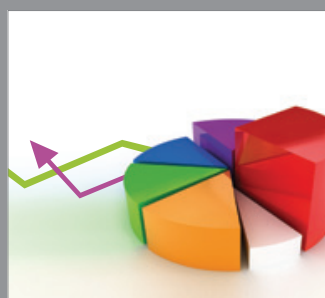

ournal of

Probability and Statistics

Promensencen
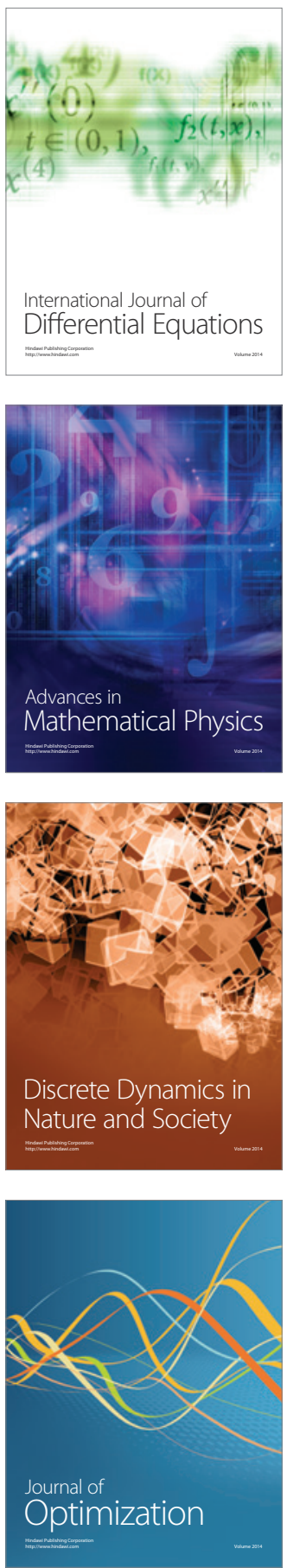